\title{
Fatores Preditores de Complicações após Radioterapia Conformacional3D em Pacientes com Adenocarcinoma de Próstata
}

doi: https://doi.org/10.32635/2176-9745.RBC.2020v66n1.530

\author{
Predictors of Complications following 3D Conformational Radiotherapy in Patients with Prostate Adenocarcinoma \\ Factores Predictores de Complicaciones después de la Radioterapia Conformacional 3D en Pacientes con Adenocarcinoma \\ de Próstata
}

\author{
Mariana Alves Ribeiro; Jorge Soares Lyra²; Ricardo Akiyoshi Nakamura³ Nathália de Oliveira Santana4; Gabriela da Silva Xavier5 \\ Geovana Logrado de Moraes ${ }^{6}$
}

Resumo

Introduçáo: $\mathrm{O}$ câncer de próstata é considerado a neoplasia maligna mais comum que acomete homens em todas as Regióes do país, à exceção do câncer de pele não melanoma. Se diagnosticado e tratado precocemente, o câncer de próstata tem alta taxa de cura; contudo, terapêuticas como a radioterapia podem gerar complicaçóes agudas que podem impactar as atividades cotidianas. Apesar das complicaçóes no pós-tratamento, a radioterapia tem sido um método bastante praticado e que apresenta resultados positivos, ocasionando melhoria da sobrevida livre de doença. Objetivo: Avaliar os principais fatores preditores de complicaçôes agudas que acometem pacientes em tratamento radioterápico para câncer de próstata. Método: Para identificaçáo de fatores preditores de complicaçóes agudas pós-radioterapia, avaliaram-se, consecutiva e prospectivamente, 208 pacientes diagnosticados com adenocarcinoma de próstata tratados com radioterapia conformacional 3D em um centro referência vinculado ao SUS entre os anos 2016 e 2017. Realizou-se ainda avaliaçáo retrospectiva de prontuários para coleta de dados adicionais. A análise estatística foi realizada por meio dos testes qui-quadrado, exato de Fisher, Anova e regressão logística ordinal. Resultados: Após análise da amostra, evidenciou-se que, entre as complicaçóes agudas, as de maior incidência foram radiodermite, cistite e enterite/retite, de forma que tais complicaçóes tiveram como fatores associados volume irradiado, tratamento prévio e sintomas prévios ao tratamento. Conclusáo: $\mathrm{O}$ estudo sugere que, apesar da existência de complicaçôes ao final do tratamento, a grande maioria é de baixa complexidade e que pacientes submetidos a procedimentos cirúrgicos prévios podem evoluir com presença de complicaçóes mais graves.

Palavras-chave: Neoplasias da Próstata; Prognóstico; Radioterapia; Toxicidade Aguda.

\begin{abstract}
Introduction: Prostate cancer is considered the most common malignancy that affects men in all regions of the country, except for non-melanoma skin cancer. If diagnosed and treated early, prostate cancer has a high cure rate; however, therapies such as radiotherapy can generate acute complications that can impact daily activities. Despite post-treatment complications, radiotherapy has been a widely practiced method and has shown positive results, leading to improved disease-free survival. Objective: To evaluate the main predictive factors for acute complications that affect patients undergoing radiotherapy for prostate cancer. Method: To identify predictive factors for acute post-radiotherapy complications, 208 patients diagnosed with prostate adenocarcinoma treated with $3 \mathrm{D}$ conformational radiotherapy were consecutively and prospectively evaluated at a referral center linked to SUS between the years 2016 and 2017. It was carried out retrospective evaluation of medical records to collect additional data. Statistical analysis was performed using the chi-square test, Fisher's exact, Anova and ordinal logistic regression. Results: After analyzing the sample, it was evidenced that among the acute complications, those with the highest incidence were radiodermatitis, cystitis, enteritis/rectitis, so that these complications had associated predictive factors as irradiated volume, previous treatment and symptoms. Conclusion: The study suggests that despite the existence of complications at the end of the treatment, the vast majority are of low complexity and that the patients submitted to previous surgical procedures can evolve with the presence of more severe complications.

Key words: Prostatic Neoplasms; Prognosis; Radiotherapy; Acute Toxicity.
\end{abstract}

\section{Resumen}

Introducción: El cáncer de próstata se considera la neoplasia maligna más común que afecta a los hombres en todas las regiones del país, con la excepción del cáncer de piel no melanoma. Si se diagnostica y trata temprano, el cáncer de próstata tiene una alta tasa de curación; sin embargo, las terapias como la radioterapia pueden generar complicaciones agudas que pueden afectar las actividades diarias. A pesar de las complicaciones posteriores al tratamiento, la radioterapia ha sido un método ampliamente practicado y ha mostrado resultados positivos, lo que lleva a una mejor supervivencia libre de enfermedad. Objetivo: Evaluar los principales predictores de complicaciones agudas que afectan a los pacientes sometidos a radioterapia para el cáncer de próstata. Método: Para identificar los factores predictivos de complicaciones agudas posteriores a la radioterapia, 208 pacientes diagnosticados con adenocarcinoma de próstata tratados con radioterapia conformacional 3D fueron evaluados consecutiva y prospectivamente en un centro de referencia vinculado al SUS entre los años 2016 y 2017. Se realizó evaluación retrospectiva de registros médicos para recopilar datos adicionales. El análisis estadístico se realizó utilizando la prueba de chi-cuadrado, exacta de Fisher, de Anova y la regresión logística ordinal. Resultados: Después de analizar la muestra, se evidenció que, entre las complicaciones agudas, las de mayor incidencia fueron radiodermatitis, cistitis, enteritis/retitis y síntomas obstructivos, por lo que estas complicaciones tenían factores predictivos asociados, como el volumen irradiado, el tratamiento previo y los síntomas. Conclusión: El estudio sugiere que a pesar de la existencia de complicaciones al final del tratamiento, la gran mayoría son de baja complejidad. Como factores predictivos encontrados, se puede mencionar el volumen irradiado, la existencia de tratamiento previo y los síntomas en la consulta inicial.

Palabra clave: Neoplasias de la Próstata; Pronóstico; Radioterapia; Toxicidad Aguda.

\footnotetext{
${ }^{1}$ Universidade Federal do Maranhão (UFMA). Imperatriz (MA), Brasil. Orcid iD: https://orcid.org/0000-0001-8085-6658

${ }^{2}$ Hospital das Clínicas de Imperatriz. Centro de Atendimento Oncoradium ${ }^{\oplus}$. UFMA. Imperatriz (MA), Brasil. Orcid iD: https://orcid.org/0000-0001-7400-829X

${ }^{3}$ Centro de Atendimento Oncoradium ${ }^{\oplus}$. UFMA. Imperatriz (MA), Brasil. Orcid iD: https://orcid.org/0000-0001-5088-9045

${ }^{4}$ UFMA. Imperatriz (MA), Brasil. Orcid iD: https://orcid.org/0000-0002-2870-3088

${ }^{5}$ UFMA. Imperatriz (MA), Brasil. Orcid iD: https://orcid.org/0000-0001- 5025-7822

${ }^{6}$ UFMA. Imperatriz (MA), Brasil. Orcid iD: https://orcid.org/0000-0001-7313-1085

Trabalho realizado no Centro de Tratamento Oncoradium ${ }^{\circledast}$.

Endereço para correspondência: Mariana Alves Ribeiro. Rua Santo Cristo, 1083, complemento 504a - Nova Imperatriz. Imperatriz (MA), Brasil. CEP 65907-040. E-mail: marianaribeiroaa@outlook.com
} 


\section{INTRODUÇÃO}

O câncer de próstata é considerado a neoplasia maligna mais comum que acomete os homens em todas as Regióes do país, à exceção do câncer de pele não melanoma ${ }^{1,2}$. Foram estimados, para cada ano do triênio 2020-2022, o surgimento de 65.840 novos casos no Brasi ${ }^{1}$. A elevada incidência está associada a fatores como idade avançada, dieta hipercalórica, hereditariedade e etnia ${ }^{2}$. O tratamento pode ser realizado por abordagem cirúrgica, radioterapia externa, hormonioterapia, crioterapia ou vigilância ativa ${ }^{2}$. Se diagnosticado e tratado precocemente, o câncer de próstata tem alta taxa de cura; contudo, terapêuticas como radioterapia podem gerar complicaçôes agudas que podem impactar as atividades cotidianas. Entende-se como complicaçóes agudas reaçôes que surgem durante o tratamento ou nos três primeiros meses seguintes ${ }^{3}$. A escolha do tratamento deveria depender do desejo do paciente e das características da doença; mas, muitas vezes, dependerá de qual profissional realizou a primeira abordagem - urologista, oncologista ou radioterapeuta ${ }^{3}$.

Apesar das complicaçóes no pós-tratamento, a radioterapia tem sido um método bastante praticado e que apresenta resultados positivos, ocasionando melhoria da sobrevida livre de doença, principalmente em pacientes com menos de 70 anos $^{2,3}$. Na radioterapia conformada tridimensional (RT3D), utiliza-se alta tecnologia para permitir melhor localização do alvo, assegurando assim maior proteção dos órgãos adjacentes. Ao ser comparado com o método convencional, nota-se que altas doses liberadas com a RT3D geram toxicidade inferior ${ }^{4,5}$. Porém, ainda se notam complicaçóes decorrentes do tratamento como radiodermite, cistite, retite, entre outros ${ }^{6,7}$.

Diante disso, o estudo visa a levantar os principais fatores preditores de toxicidade aguda no tratamento radioterápico em pacientes com câncer de próstata. De forma que, após a análise, seja possível elencar fatores com significância que possam contribuir para um manejo médico mais eficaz para assegurar menos complicações.

\section{MÉTODO}

Trata-se de um trabalho retrospectivo, descritivo e unicêntrico, realizado entre janeiro de 2016 e dezembro de 2017 no Centro de Tratamento Oncoradium. Foram avaliados 208 prontuários consecutivos de uma única instituição vinculada ao Sistema nico de Saúde (SUS) correspondentes a pacientes com adenocarcinoma de próstata diagnosticados por biópsia transretal. Foram excluídos da análise pacientes que vieram a óbito, apresentaram metástase ou não possuíam informações suficientes nos prontuários.
O levantamento de dados foi realizado por meio da análise dos prontuários médicos que continham identificação do paciente, idade, comorbidades, histórico de câncer na família, presença de tratamento prévio, sintomas na primeira consulta com o radioterapeuta, estadiamento de acordo com o Sistema TNM de Classificação dos Tumores Malignos, Gleason, antígeno prostático específico (PSA) total, ficha com dados técnicos sobre a radioterapia, finalidade do tratamento e presença de complicaçôes agudas. A identificação dos prontuários ocorreu mediante Código C61 da Classificação Internacional de Doenças e Problemas Relacionados à Saúde - 10aedição (CID-10), presente em todos os prontuários, e os pacientes foram estadiados segundo o Sistema TNM da American Joint Committee of Cancer (AJCC), $7^{\mathrm{a}} \mathrm{ed}$. Para fins do estudo, os pacientes foram reestadiados de acordo com a atualização do AJCC $8^{\mathrm{a}} \mathrm{ed}^{8}$. O projeto foi aprovado pelo Comitê de Ética e Bioética da Faculdade de Imperatriz, sob o número de parecer 09422017.

Com relação ao tratamento, o volume-alvo de planejamento (PTV) foi calculado pelo Departamento de Física Médica do estabelecimento, utilizando o sistema Eclipse Planning (versão 10.0, Varian Medical Systems, Palo Alto, $\mathrm{CA}^{\odot}$ ), do programa External Beam Planning, por meio da tomografia computadorizada, acrescido de margens de $10 \mathrm{~mm}$ em todas as direçóes, exceto no reto com $6 \mathrm{~mm}$, conforme exposto na Figura 1. Todos os pacientes do presente estudo foram submetidos à RT3D, método que emite feixes de fótons lineares com energia de 6MV, utilizando-se usualmente quatro campos de irradiação, por intermédio do Acelerador Linear Clinac $6 E X^{\circledR}$ do fabricante Varian Medical Systems Brasil Ltda ${ }^{\circledR}$, em decúbito dorsal, com a bexiga cheia e apoio sob os pés, sendo feita a irradiação de 1,8 a 2 Gy por dia, cinco dias por semana, com dose de radiaçáo determinada a critério do radioterapeuta. As irradiaçóes foram divididas em duas fases, sendo a primeira fase com irradiação sobre

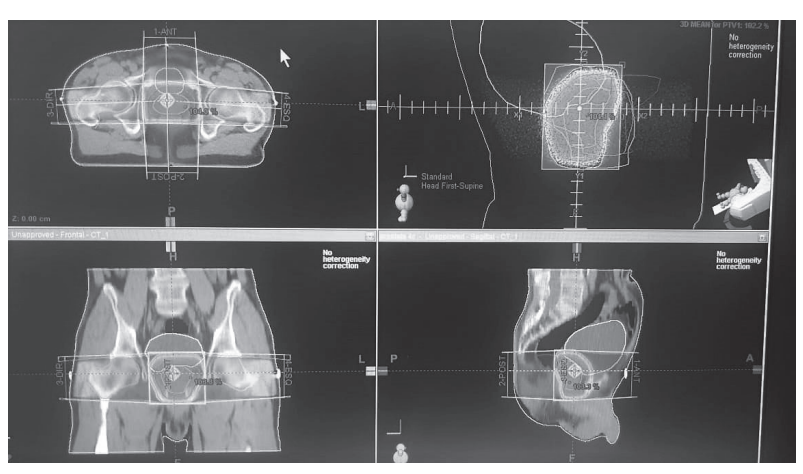

Figura 1. Captura de tela mostrando cálculo do volume-alvo de planejamento da próstata utilizando o sistema Eclipse Planning 
próstata/leito prostático e vesículas seminais (VVSS)/leito VVSS correspondendo ao PTV1 e a segunda fase sobre a próstata/leito prostático, correspondendo ao PTV2, respeitando sempre o limite de dose de irradiação aceitável para que não cause danos significativos, essas restrições de doses dizem respeito, segundo a Quantitative Analysis of Normal Tissue Effects in the Clinic (Quantec) ${ }^{9}$, à bexiga: 80 $\mathrm{Gy}<15 \%, 75 \mathrm{~Gy}<25 \%, 70 \mathrm{~Gy}<35 \%$ e $65 \mathrm{~Gy}<50 \%$; ao reto: $75 \mathrm{~Gy}<15 \%, 70 \mathrm{~Gy}<20 \%, 65 \mathrm{~Gy}<25 \%, 60 \mathrm{~Gy}<35 \%$ e $50 \mathrm{~Gy}<50 \%$; e às cabeças femorais: $50 \mathrm{~Gy}<5 \%$.

Durante o tratamento, os pacientes realizaram consultas semanais nas quais foram investigadas as eventuais queixas urinárias e as possíveis complicaçôes gastrointestinais agudas decorrentes da terapêutica. As complicações foram classificadas segundo o Common Terminology Criteria for Adverse Events (CTCAE) ${ }^{10}$ v5.0, de acordo com as queixas apresentadas pelos pacientes
(Quadro 1). Outros critérios avaliados estáo presentes nas Tabelas 1 e 2.

Após a identificação dos pacientes, os dados foram registrados e, em seguida, tabulados em uma planilha eletrônica confeccionada pelo software Excel (Microsoft Office Excel 11.0/2007 - Microsoft Corporation ${ }^{\oplus}$ ) e, posteriormente, processados com auxílio do software Minitab 18 - SPSS. A análise se dividiu em exploratória com tabulação e gráfico de todas as variáveis e testes de hipótese aplicados via Minitab 18 -SPSS; as associaçóes entre as variáveis foram verificadas pelo teste do qui-quadrado, assim como pelos testes exato de Fisher e de análise de variância Anova. Em conjunto, foi utilizado o modelo de regressão logística ordinal, amplamente utilizado na medicina, o qual visa a indicar qual a probabilidade de as variáveis preditoras influenciarem na ocorrência da variável resposta com níveis de ordenação.

Quadro 1. Complicações urinárias e gastrointestinais (CTCAE v5.0)

\begin{tabular}{|c|c|c|c|c|c|}
\hline Complicações & Grau 1 & Grau 2 & Grau 3 & Grau 4 & Grau 5 \\
\hline Radiodermite & $\begin{array}{l}\text { Eritema franco, } \\
\text { com ressecamento } \\
\text { ou descamação }\end{array}$ & $\begin{array}{l}\text { Eritema com } \\
\text { edema moderado, } \\
\text { descamação } \\
\text { úmida } \\
\text { principalmente em } \\
\text { dobras cutâneas }\end{array}$ & $\begin{array}{l}\text { Descamação } \\
\text { úmida em } \\
\text { áreas além } \\
\text { das dobras } \\
\text { cutâneas, } \\
\text { sangramento } \\
\text { induzido por } \\
\text { trauma menor } \\
\text { ou abrasão }\end{array}$ & $\begin{array}{l}\text { Necrose } \\
\text { de pele ou } \\
\text { ulceração } \\
\text { acometendo } \\
\text { a derme } \\
\text { Sangramento } \\
\text { espontâneo } \\
\text { do sítio } \\
\text { envolvido com } \\
\text { risco de vida } \\
\text { Indicação de } \\
\text { enxerto de } \\
\text { pele }\end{array}$ & Morte \\
\hline Cistite & $\begin{array}{l}\text { Hematúria } \\
\text { microscópica, } \\
\text { aumento na } \\
\text { frequência e } \\
\text { urgência urinária, } \\
\text { disúria leve ou } \\
\text { noctúria }\end{array}$ & $\begin{array}{l}\text { Moderada } \\
\text { hematúria, } \\
\text { disúria e noctúria } \\
\text { Aumento } \\
\text { moderado na } \\
\text { frequência, } \\
\text { incontinência e } \\
\text { urgência urinária } \\
\text { Colocação } \\
\text { de cateter ou } \\
\text { irrigação da } \\
\text { bexiga indicado }\end{array}$ & $\begin{array}{l}\text { Hematúria } \\
\text { severa com } \\
\text { indicação de } \\
\text { transfusão, } \\
\text { medicamentos } \\
\text { IV ou } \\
\text { hospitalização, } \\
\text { indicação de } \\
\text { intervenção } \\
\text { invasiva eletiva }\end{array}$ & $\begin{array}{l}\text { Apresenta } \\
\text { risco de vida, } \\
\text { urgência em } \\
\text { indicação } \\
\text { invasiva }\end{array}$ & Morte \\
\hline Disúria & Presente & - & - & - & - \\
\hline Retite & $\begin{array}{l}\text { Desconforto retal } \\
\text { sem indicação de } \\
\text { intervenção }\end{array}$ & $\begin{array}{l}\text { Desconforto } \\
\text { retal, presença } \\
\text { de sangue ou } \\
\text { muco durante } \\
\text { as evacuações, } \\
\text { intervenção } \\
\text { médica indicada }\end{array}$ & $\begin{array}{l}\text { Sintomas } \\
\text { graves com } \\
\text { urgência ou } \\
\text { incontinência } \\
\text { fecal } \\
\text { necessitando } \\
\text { de fraldas }\end{array}$ & $\begin{array}{l}\text { Perfuração, } \\
\text { sangramento } \\
\text { ou necrose } \\
\text { com risco de } \\
\text { morte que } \\
\text { necessite de } \\
\text { intervenção } \\
\text { cirúrgica }\end{array}$ & Morte \\
\hline
\end{tabular}

Legenda: $\mathrm{CTCAE}=$ Common Terminology Criteria for Adverse Event. 
A função utilizada para ajustamento das equaçôes foi o modelo Logito (logit). O nível de significância atribuído nos testes é de 5\%, com intervalo de confiança de $95 \%$ e erro amostral calculado de $5 \%$.

\section{RESULTADOS}

Foram avaliados 208 pacientes com média de idade de 72 anos. $\mathrm{Na}$ análise referente aos hábitos e à existência de comorbidades $(p=0,156)$, evidenciou-se que 131 pacientes (63\%) relataram ser ex-tabagistas, 53 pacientes (25\%) ex-etilista, 109 pacientes (52\%) tinham hipertensão arterial sistêmica e 18 pacientes (9\%) diabetes mellitus, e 87 pacientes $(41,8 \%)$ afirmaram histórico de câncer na família. Constatou-se que 126 pacientes $(56 \%)$ relataram sintomas na consulta inicial com o radioterapeuta pré-tratamento $(p=0,026)$, sendo que, destes, os mais comuns foram urgeincontinência urinária moderada/grave, presente em 41 pacientes $(20 \%)$, seguido de urgeincontinência urinária leve em 30 pacientes $(14 \%)$, gotejamento terminal em 26 pacientes (13\%) e disúria leve em 22 pacientes (11\%). Após análise, evidenciou-se que 114 pacientes $(54,8 \%)$ realizaram um tratamento prévio e 63 pacientes $(30,3 \%)$ dois tratamentos, sendo os mais realizados a hormonioterapia neoadjuvante presente em 129 pacientes $(62 \%)$, seguida da prostatectomia radical em 76 pacientes (37\%), conforme exposto na Tabela 1.

A maioria das complicaçóes agudas encontradas na amostra foi classificada como baixo risco segundo o CTCAE v5.0, sendo as de maior prevalência radiodermite, cistite, enterite/retite e sintomas obstrutivos, conforme verificado na Tabela 2.

Constatou-se que 131 pacientes $(62,98 \%)$ realizaram radioterapia de caráter radical/curativa $(p=0,979)$, de forma que a finalidade do tratamento radioterápico aplicado não influencie diretamente na existência de complicaçôes após o tratamento. Verificou-se que 22 pacientes (11\%) apresentaram recidiva bioquímica, porém se constatou que não há influência direta no surgimento de complicaçôes pós radioterapia $(p=0,195)$.

No que diz respeito à dose de irradiação recebida durante o tratamento, cerca de 170 pacientes $(81,73 \%)$ receberam uma média de dose inicial de 54 Gy em PTV1, e 112 pacientes $(53,85 \%)$, doses acima de 70 Gy como dose de complementação em PTV2.

Para avaliação de complicaçóes relacionadas com o volume médio irradiado em cada estrutura (PTV), a comparação resultante do teste Anova demonstrou que pacientes que irradiaram acima de $300 \mathrm{~cm}^{3} \mathrm{em}$ bexiga $(p=0,059)$ apresentaram maior grau de complicação, sendo que $19,7 \%$ evoluíram com cistite grau 1 e 14,4\% com cistite grau 2. Para os que irradiaram acima de 50 $\mathrm{cm}^{3}$ em reto $(p=0,573), 44,2 \%$ evoluíram com enterite/ retite grau 1 e $2,4 \%$ com enterite/retite grau 2 e $38 \%$ dos pacientes que irradiaram entre $100 \mathrm{a} 200 \mathrm{~cm}^{3} \mathrm{em}$ próstata + VVSS $(p=0,001)$ apresentaram cistite grau $1 \mathrm{e}$ $26,4 \%$ cistite grau 2 . Ao passo que $23,56 \%$ dos pacientes que irradiaram entre $100 \mathrm{a} 200 \mathrm{~cm}^{3}$ em leito prostático + VVSS $(p<0,001)$ apresentaram cistite grau 1 e $44,7 \%$ dos pacientes que irradiaram próstata $(p=0,019)$ apresentaram cistite grau 2.

\section{DISCUSSÃO}

Conforme observado nos resultados, a idade média foi de 72 anos (43-87), o que corrobora as demais literaturas, visto que a idade média para a ocorrência do câncer de próstata encontra-se acima da $6^{a}$ década de vida ${ }^{5,11}$. A literatura considera hipertensão arterial, diabetes mellitus $\mathrm{e}$ vasculites como fatores predisponentes para toxicidade da radioterapia aumentad $\mathrm{a}^{11}$. No entanto, no presente estudo, as comorbidades não influenciaram para o surgimento de complicaçôes agudas $(p=0,156)$.

Nakamura et al. ${ }^{5}$ constataram maior incidência de adenocarcinoma de próstata em estágios mais iniciais ( $\leq \mathrm{T} 2 \mathrm{a})$; entretanto, na presente análise, o estádio T2bT2c foi o mais prevalente. A situaçáo provavelmente ocorreu pela diferença da populaçáo estudada, uma vez que o presente estudo foi realizado em uma Região onde o índice de subdesenvolvimento é significativo e, possivelmente, por serem menos instruídos, os pacientes procuram atendimento de forma mais $\operatorname{tardia}^{12}$. Na presente amostra, o estágio do câncer náo influenciou diretamente a existência de complicaçóes agudas após o tratamento $(p=0,522)$. Na presente análise, os valores de Gleason e PSA não foram significativos para o surgimento de complicaçóes, condizente com estudo conduzido por Afonso-João et al. . .

Segundo o estadiamento de risco D'Amico, James et al. ${ }^{13}$ evidenciaram maior prevalência do risco intermediário (48\%), seguido do alto risco (32\%). Na presente série, o grupo mais prevalente foi de alto risco $(60 \%)$, em seguida o de risco intermediário (27\%), notando-se uma pequena discrepância referente às literaturas vigentes, o que corrobora a sugestão de que os pacientes analisados iniciaram o tratamento mais tardiamente quando comparados aos demais. Foi constatado também que $48,5 \%$ dos pacientes apresentaram, no mínimo, uma queixa durante a consulta inicial com o radioterapeuta $(p=0,026)$, e que a existência de tratamento prévio pode influenciar no surgimento de complicações agudas após radioterapia $(p=0,006)$, de modo que os procedimentos cirúrgicos como a prostatectomia radical em conjunto 
Tabela 1. Características do paciente e do tratamento RT3D

\begin{tabular}{|c|c|c|c|}
\hline Variável & Categoria & Número de pacientes & $\%$ \\
\hline Idade & $\begin{array}{l}\text { Até } 50 \text { anos } \\
\text { Entre } 50-70 \\
\text { Entre } 70-80 \\
>80\end{array}$ & $\begin{array}{c}2 \\
104 \\
108 \\
8\end{array}$ & $\begin{array}{c}1 \\
43 \\
52 \\
4\end{array}$ \\
\hline Comorbidades & $\begin{array}{l}\text { Sim } \\
\text { Não }\end{array}$ & $\begin{array}{c}186 \\
22\end{array}$ & $\begin{array}{l}89 \\
11\end{array}$ \\
\hline Gleason & $\begin{array}{l}\leq 6 \\
7 \\
\geq 8\end{array}$ & $\begin{array}{l}77 \\
90 \\
39\end{array}$ & $\begin{array}{l}37 \\
43 \\
19\end{array}$ \\
\hline PSA total & $\begin{array}{l}<4 \\
4<10 \\
10<20 \\
>20\end{array}$ & $\begin{array}{l}65 \\
47 \\
38 \\
57\end{array}$ & $\begin{array}{l}31 \\
23 \\
18 \\
26\end{array}$ \\
\hline Estadiamento T & $\begin{array}{l}\leq \mathrm{T} 2 \mathrm{a} \\
\mathrm{T} 2 \mathrm{~b}-\mathrm{T} 2 \mathrm{c} \\
\geq \mathrm{T} 3\end{array}$ & $\begin{array}{l}56 \\
95 \\
57\end{array}$ & $\begin{array}{c}26,91 \\
45,6 \\
27,33\end{array}$ \\
\hline Risco & $\begin{array}{l}\text { Baixo } \\
\text { Intermediário } \\
\text { Alto } \\
\text { Muito alto }\end{array}$ & $\begin{array}{c}11 \\
56 \\
124 \\
10\end{array}$ & $\begin{array}{c}5 \\
27 \\
60 \\
5\end{array}$ \\
\hline Sintomas na primeira consulta & $\begin{array}{l}\text { Nenhum } \\
\text { Entre 1-3 } \\
\geq 4\end{array}$ & $\begin{array}{c}92 \\
101 \\
15\end{array}$ & $\begin{array}{c}44 \\
48,5 \\
7,5\end{array}$ \\
\hline Tipo de tratamento prévio & $\begin{array}{l}\text { Hormonioterapia neoadjuvante } \\
\text { Prostatectomia radical } \\
\text { Orquiectomia } \\
\text { Ressecção transuretral }\end{array}$ & $\begin{array}{l}129 \\
76 \\
13 \\
20\end{array}$ & $\begin{array}{c}62 \\
37 \\
6 \\
10\end{array}$ \\
\hline Recidiva bioquímica & $\begin{array}{l}\text { Sim } \\
\text { Não }\end{array}$ & $\begin{array}{l}22 \\
18\end{array}$ & $\begin{array}{l}11 \\
88\end{array}$ \\
\hline Caráter da radioterapia & $\begin{array}{l}\text { Radical/curativo } \\
\text { Resgate/adjuvante }\end{array}$ & $\begin{array}{c}131 \\
75\end{array}$ & $\begin{array}{l}62,98 \\
36,06\end{array}$ \\
\hline Número de sessões & $\begin{array}{l}36-38 \\
39\end{array}$ & $\begin{array}{c}101 \\
83\end{array}$ & $\begin{array}{l}49 \\
40\end{array}$ \\
\hline Tempo de radioterapia & $\begin{array}{l}1-2 \text { meses } \\
2-3 \text { meses }\end{array}$ & $\begin{array}{c}19 \\
187\end{array}$ & $\begin{array}{c}9 \\
90\end{array}$ \\
\hline Irradiação pelve & $\begin{array}{l}\text { Sim } \\
\text { Não }\end{array}$ & $\begin{array}{c}28 \\
180\end{array}$ & $\begin{array}{l}12,97 \\
87,03\end{array}$ \\
\hline Irradiação VVSS/ leito VVSS & $\begin{array}{l}\text { Sim } \\
\text { Não }\end{array}$ & $\begin{array}{c}200 \\
8\end{array}$ & $\begin{array}{c}96,15 \\
3,85\end{array}$ \\
\hline $\begin{array}{l}\text { Irradiação próstata/ } \\
\text { Leito prostático }\end{array}$ & Sim & 208 & 100 \\
\hline Dose (Gy) & $\begin{array}{l}\text { Inicial entre } 50-60 \\
\text { Inicial } \geq 60 \\
\text { boost entre } 10-20 \\
\text { boost }>20\end{array}$ & $\begin{array}{c}170 \\
30 \\
74 \\
112\end{array}$ & $\begin{array}{l}81,73 \\
14,42 \\
35,78 \\
53,85\end{array}$ \\
\hline Principais sintomas & $\begin{array}{l}\text { Disúria leve } \\
\text { Constipação } \\
\text { Sintomas obstrutivos } \\
\text { Polaciúria }\end{array}$ & $\begin{array}{l}105 \\
49 \\
30 \\
25\end{array}$ & $\begin{array}{c}50 \\
24 \\
14 \\
1\end{array}$ \\
\hline
\end{tabular}

Legendas: PSA=antígeno prostático específico; RT3D=radioterapia conformada tridimensional; VVSS=vesículas seminais; Gy=gray. 
Ribeiro MA, Lyra JS, Nakamura RA, Santana NO, Xavier GS, Moraes GL

Tabela 2. Complicações agudas pós-radioterapia (CTCAE v5.0)

\begin{tabular}{lcc}
\hline \multicolumn{1}{c}{ Complicações } & $\begin{array}{c}\text { Número de } \\
\text { pacientes }\end{array}$ & \multicolumn{1}{c}{$\%$} \\
\hline Radiodermite grau 1 & 121 & 58,2 \\
Radiodermite graus 2-3 & 14 & 6,7 \\
Cistite graus 1-2 & 135 & 64,9 \\
Enterite/Retite graus 1-2 & 123 & 59,2 \\
Enterite/Retite grau 3 & 7 & 3,4 \\
Sintomas obstrutivos & 12 & 5,7 \\
Sem complicações & 40 & 19,2 \\
\hline
\end{tabular}

Legenda: CTCAE=Common Terminology Criteria for Adverse Events.

com a RTU e a orquiectomia foram os que apresentaram maior efeito negativo sobre o surgimento de complicaçóes quando comparados com as demais terapêuticas prévias, como a hormonioterapia, na qual menos da metade dos pacientes (42,3\%) evoluiu com complicaçóes leves como radiodermite grau $1(p=0,997)$, de acordo com o exposto na Tabela 3.

Tabela 3. Distribuição dos pacientes segundo o tratamento prévio de acordo com a complicação apresentada após o tratamento

\begin{tabular}{|c|c|c|c|}
\hline \multicolumn{4}{|c|}{ Orquiectomia } \\
\hline Cistite & $\begin{array}{l}\text { Grau } 1 \\
\text { Grau } 2\end{array}$ & $\begin{array}{l}36,5 \\
24,5\end{array}$ & 0,463 \\
\hline Disúria & $\begin{array}{l}\text { Grau } 1 \\
\text { Grau } 2\end{array}$ & $\begin{array}{l}0,5 \\
0,5\end{array}$ & 0,062 \\
\hline Enterite & $\begin{array}{l}\text { Grau } 1 \\
\text { Grau } 2 \\
\text { Grau } 3\end{array}$ & $\begin{array}{l}2,4 \\
7,7 \\
3,4\end{array}$ & 0,720 \\
\hline Radiodermite & $\begin{array}{l}\text { Grau } 1 \\
\text { Grau } 2\end{array}$ & $\begin{array}{l}2,4 \\
1,0\end{array}$ & 0,233 \\
\hline \multicolumn{4}{|c|}{ Prostatectomia } \\
\hline Cistite & $\begin{array}{l}\text { Grau } 1 \\
\text { Grau } 2\end{array}$ & $\begin{array}{l}12,5 \\
11,5\end{array}$ & 0,487 \\
\hline Enterite & $\begin{array}{l}\text { Grau } 1 \\
\text { Grau } 2 \\
\text { Grau } 3\end{array}$ & $\begin{array}{c}14,4 \\
5,8 \\
1,0\end{array}$ & 0,046 \\
\hline Radiodermite & $\begin{array}{l}\text { Grau } 1 \\
\text { Grau } 2 \\
\text { Grau } 3\end{array}$ & $\begin{array}{c}23,6 \\
1,4 \\
0,5\end{array}$ & 0,565 \\
\hline \multicolumn{4}{|c|}{ Ressecção transuretral } \\
\hline Cistite & $\begin{array}{l}\text { Grau } 1 \\
\text { Grau } 2\end{array}$ & $\begin{array}{l}3,4 \\
5,3\end{array}$ & 0,005 \\
\hline Enterite & $\begin{array}{l}\text { Grau } 1 \\
\text { Grau } 2 \\
\text { Grau } 3\end{array}$ & $\begin{array}{l}3,8 \\
1,9 \\
1,4\end{array}$ & 0,003 \\
\hline Radiodermite & $\begin{array}{l}\text { Grau } 1 \\
\text { Grau } 2\end{array}$ & $\begin{array}{l}7,2 \\
0,5\end{array}$ & 0,418 \\
\hline
\end{tabular}

Segundo Bedini et al..$^{14}$, pacientes submetidos à cirurgia abdominal pré-tratamento apresentam resposta diferente à radioterapia com maiores complicaçóes agudas, em razão da maior radiossensibilidade presente nas células, somada a complicações que podem ocorrer durante a cirurgia. Neste mesmo estudo, $15 \%$ dos pacientes submetidos à prostatectomia radical apresentaram toxicidade retal aguda grau 2 e 56,3\% mostraram valores de toxicidade múltipla (>3 complicaçóes). No estudo Protect Trial ${ }^{15}$, a principal complicação pós-operatória encontrada foi incontinência urinária representada por $1 \%$ ao início do estudo e $46 \%$ aos seis meses. Pode-se inferir que os dados encontrados são condizentes com o que está presente na literatura.

O tempo de duração do tratamento radioterápico foi entre dois e três meses. Segundo análise estatística observada, infere-se que o tempo de tratamento influencia na existência de complicaçôes, indicando que quanto maior o número de sessóes e o tempo de tratamento, maior o acumulativo de irradiaçáo que o paciente irá receber e, consequentemente, estará propenso a desenvolver maior número de efeitos adversos $(p=0,016)$. A duração da radioterapia encontrada condiz com dados vigentes na literatura ${ }^{15}$. Nakamura et al. ${ }^{5}$ relataram em seu estudo um tempo de tratamento médio de 71 dias (2, 3 meses).

Quanto às complicaçôes, observou-se que a maioria seguiu um curso de menor complexidade, segundo exposto na Tabela 2. Foram avaliados no estudo apenas complicaçóes decorrentes de toxicidade aguda, definida como eventos adversos ocorridos durante a radioterapia ou nos três primeiros meses seguintes ${ }^{16,17}$. Dias ${ }^{17}$ encontrou que $35,9 \%$ dos pacientes apresentaram toxicidade retal aguda grau 1 , enquanto $22,2 \%$ dos pacientes apresentaram grau $\geq 2$. Gill et al. ${ }^{16}$ verificaram que a toxicidade urinária grau 1 esteve presente em 35\% dos pacientes; grau $2 \mathrm{em}$ $38 \%$; e grau $3 \mathrm{em} 23 \%$. No que se refere ao acometimento gastrointestinal, a toxicidade grau 1 esteve presente em $35 \%$ e a grau 2, em $19 \%$. Segundo estudo conduzido por Dias $^{17}, 40,3 \%$ dos pacientes apresentaram complicação urinária aguda grau 1 e $36,7 \% \geq$ grau 2 . Em relação às complicaçóes gastrointestinais, ainda neste mesmo estudo, 17,4\% apresentaram complicação grau $1 \mathrm{e}$ $11,9 \% \geq$ grau 2 . Pode-se afirmar que os dados do atual estudo corroboram as literaturas existentes, tal fato se relaciona à técnica do tratamento empregada que busca uma precisão cada vez maior de irradiação no órgão-alvo. Estudos apontam que a presença de complicaçóes agudas $\geq$ grau 2 constitui fator prognóstico para o surgimento de complicaçóes tardias ${ }^{18}$ de forma que, entre os pacientes que desenvolveram sintomas agudos durante o tratamento, a incidência de toxicidade tardia foi de $35 \%$ em dez anos ${ }^{19}$.

Em se tratando da existência de complicaçóes relacionadas com o volume de alvos, o estudo revelou 
que quanto maior o volume médio irradiado, maior o número de complicaçóes que o paciente está sujeito a apresentar. Evidenciou-se que o volume médio em leito prostático + VVSS foi maior para indivíduos que apresentaram quatro complicaçóes após o tratamento em relação aos que apresentaram três ou menos complicaçóes. De forma que pacientes com irradiação na bexiga $>300$ $\mathrm{cm}^{3}$ evoluíram com mais complicaçóes agudas. Tal análise condiz com a literatura; segundo Monti et al. ${ }^{20}$, doses $>70$ Gy em 30\% do volume da bexiga implicam em maior toxicidade aguda.

\section{CONCLUSÃO}

Apesar da existência de complicaçôes ao final do tratamento, nota-se que a grande maioria é de baixa complexidade; ou seja, complicaçóes agudas graus 1-2, o que reforça a prática do método radioterápico como uma modalidade que apresenta resultados satisfatórios. Foi possível constatar que quanto maior o volume irradiado nas áreas observadas associado à existência de tratamento prévio e sintomas na consulta inicial, maior será a probabilidade de um paciente apresentar complicaçóes agudas após o tratamento. Ao final do estudo, é possível sugerir que pacientes submetidos a procedimentos cirúrgicos prévios, muitas vezes de forma desnecessária, possam evoluir com presença de complicaçóes mais graves e isso tem como reflexo o aumento da morbidade do método.

\section{CONTRIBUIÇÕES}

Todos os autores contribuíram substancialmente na concepção e no planejamento do estudo, na obtenção, análise e/ ou interpretação dos dados, assim como na redação e/ou revisão crítica e aprovaram a versão final a ser publicada.

\section{DECLARAÇÃO DE CONFLITOS DE INTERESSE}

Nada a declarar.

\section{FONTES DE FINANCIAMENTO}

Não há.

\section{REFERÊNCIAS}

1. Instituto Nacional de Câncer José Alencar Gomes da Silva. Estimativa 2020: incidência de câncer no Brasil. Rio de Janeiro: INCA; 2019

2. Balbontín F, Pizzi P, Canals A, et al. Low dose rate brachytherapy in low and middle risk prostate cancer: results and impact on quality of life with 5 year follow up. Arch Esp Urol [Internet]. 2017 [cited 2017 Dec 7];70(10):824-32. Available from:http://aeurologia.com/ articulo_prod.php?id_art=7175333969205

3. Martin NE, Mucci LA, Loda M, et al. Prognostic determinants in prostate cancer. Cancer J. 2011;17(6):429-37. doi: https://doi.org/10.1097/ PPO.0b013e31823b042c

4. Poli APDF, Dias RS, Giodarni AJ, et al. Strategies to evaluate the impact of rectal volume on prostate motion during three-dimensional conformal radiotherapy for prostate cancer. Radiol Bras. 2016;49(1):17-20. doi: https://doi.org/10.1590/0100-3984.2015.0005

5. Nakamura RA, Monti CR, Trevisan FA, et al. Radioterapia externa conformada 3D para o carcinoma de próstata: experiência do Instituto do Radium de Campinas com 285 pacientes. Radiol Bras. 2009;42(2):75-82. doi: https://doi.org/10.1590/S0100-39842009000200004

6. Yu JB, Hamstra D. Point: which treatment modality for localized prostate cancer yields superior quality of life: radiotherapy or prostatectomy?. Oncol J [Internet]. 2017 [cited 2017 Dec 6];31(11). Available from: http:// www.cancernetwork.com/prostate-cancer/point-whichtreatment-modality-localizedprostate-cancer-yieldssuperior-quality-life-radiotherapy

7. Afonso-João D, Pacheco-Figueiredo L, Antunes-Lopes $\mathrm{T}$, et al. Cumulative incidence and predictive factors of radiation cystitis in patients with localized prostate cancer. Actas Urol Esp. 2018;42(4):256-61. doi: https:// doi.org/10.1016/j.acuro.2017.06.009

8. American Cancer Society [Internet]. Atlanta: ACS; c2020. Prostate cancer stages and other ways to assess risk. [revised 2019 Aug 1; cited 2020 Mar 26]. Available from: https://www.cancer.org/cancer/prostate-cancer/ detection-diagnosis-staging/staging.html

9. Radiation Oncology/Toxicity/QUANTEC [wiki on the Internet]. [place unknown]: Wikibooks. 2015 Sept 23 - [cited 2018 May 15]. Available from: https:// en.wikibooks.org/wiki/Radiation_Oncology/Toxicity/ QUANTEC.

10. Diane MF Savarese, MD. Common terminology criteria for adverse events. Waltham, MA: UpToDate Inc; 2017 Nov. [cited 2018 May 14]. Available from: https://www. uptodate.com/contents/common-terminology-criteriafor-adverse-events

11. Radiation Oncology/Toxicity/RTOG [wiki on the Internet]. [place unknown]: Wikibooks. 2020 March 20 [cited 2018 May 12]. Available from:https://en.wikibooks. org/wiki/Radiation_Oncology/Toxicity/RTOG

12. Ribeiro PVF, Silva RR, Santos KKDAT, et al. Análise clínica e epidemiológica de 348 casos de adenocarcinoma prostático atendidos em um centro oncológico de referência no Maranhão, Brasil. Rev Bras Cancerol. 2013;59(4):513-21. 
13. James M, Mclean G, Williams S, et al. Complications of curative radiation treatment for early prostate cancer. Asia Pac J Clin Oncol. 2018;14(5):e392-e398. doi: https:// doi.org/10.1111/ajco.12839

14. Bedini N, Cicchetti A, Palorini F, et al. Evaluation of mediators associated with the inflammatory response in prostate cancer patients undergoing radiotherapy. Dis Markers. 2018;2018:ID 9128128. doi: https://doi. org/10.1155/2018/9128128

15. Donovan JL, Hamdy FC, Lane JA, et al. Patient-Reported outcomes after monitoring, surgery, or radiotherapy for prostate cancer. N Engl J Med.2016;375(15):1425-37. doi: https://doi.org/10.1056/NEJMoa1606221

16. Gill S, Thomas J, Fox C, et al. Acute toxicity in prostate cancer patients treated with and without image-guided radiotherapy. Radiat Oncol. 2011;6(145). doi: https:// doi.org/10.1186/1748-717X-6-145

17. Dias RS. Definição de margens para órgãos de risco e sua relaçáo com complicações agudas e tardias da radioterapia conformacionada em câncer de próstata. [tese]. São Paulo: Universidade Federal de São Paulo, Escola Paulista de Medicina; 2011.

18. Delobel JB, Gnep K, Ospina JD, et al. Nomogram to predict rectal toxicity following prostate cancer radiotherapy. PlosOne. 2017;12(6):e0179845. doi: https://doi.org/10.1371/journal.pone.0179845

19. Zelefsky MJ, Levin EJ, Hunt M, et al. Incidence of late rectal and urinary toxicities after three-dimensional conformal radiotherapy and intensity-modulated radiotherapy for localized prostate cancer. Int J Radiat Oncol Biol Phys. 2008;70(4):1124-9. doi: https://doi. org/10.1016/j.ijrobp.2007.11.044

20. Monti CR, Nakamura RA, Ferrigno R, et al. Salvage conformal radiotherapy for biochemical recurrent prostate cancer after radical prostatectomy. Int Braz J Urol. 2006;32(4):416-26. doi: https://doi.org/10.1590/ S1677-55382006000400006 